\title{
Density of states and magneto-optical conductivity of graphene in a perpendicular magnetic field
}

\author{
C. H. Yang, ${ }^{1,2}$ F. M. Peeters, ${ }^{2, *}$ and W. $\mathrm{Xu}^{3,4}$ \\ ${ }^{1}$ College of Math and Physics, Nanjing University of Information Science and Technology, Nanjing 210044, China \\ ${ }^{2}$ Departement Fysica, Universiteit Antwerpen, Groenenborgerlaan 171, B-2020 Antwerpen, Belgium \\ ${ }^{3}$ Institute of Solid State Physics, Chinese Academy of Sciences, Hefei 230031, China \\ ${ }^{4}$ Department of Physics, Yunnan University, Kunming 610015, China
}

(Received 24 August 2010; published 18 November 2010)

\begin{abstract}
The density of states (DOS) and the optical conductivity of graphene is calculated in the presence of a perpendicular magnetic field and where scattering on charged and short-range impurities is included. The standard Kubo formula is employed where the self-energy induced by impurity scattering and the Green's function are calculated self-consistently including inter-Landau level (LL) coupling and screening effects. It is found that the scattering from those two types of impurities results in a symmetric LL broadening and asymmetric inter-LL coupling renormalizes the LL positions to lower energy. The peak position and intensity of the magneto-optical conductivity depends on the filling factor and the broadened DOS. Good agreement is found with recent cyclotron resonance measurements.
\end{abstract}

DOI: 10.1103/PhysRevB.82.205428

PACS number(s): 72.80.Vp, 78.67.Wj, 72.10.-d

\section{INTRODUCTION}

Graphene, one atomic thick sheet of carbon, shows several exotic features with a linear energy dispersion around the two nodal points $K$ and $K^{\prime}$ in the Brillouin zone. This differs strongly from a conventional two-dimensional electron gas (2DEG) that typically exhibits a parabolic energy spectrum (e.g., inversion layer at a Si surface, GaAs/GaAlAs semiconductor heterostructure). When a magnetic field is applied perpendicular to the 2D plane of graphene, the electron energy is quantized into Landau levels (LL) with an energy spectrum $E_{n}= \pm \sqrt{|n|} \hbar \omega_{B}$, where $n=0, \pm 1, \ldots$ and $\omega_{B}$ $=v_{F} \sqrt{2 e B / \hbar}$ with $v_{F}$ the Fermi velocity. The LLs are nonequidistant and the frequency $\omega_{B}$ is proportional to $\sqrt{B}$ rather than the usual linear dependence on $B$. Interestingly, the Dirac point in the energy spectrum at $k=0$ now gives an extra LL with $E_{0}=0$ shared by both electrons and holes without an energy gap. These remarkable features of the energy spectrum of graphene have resulted in unique physical properties such as the anomalous integer quantum Hall effect and a finite conductivity at the Dirac point. ${ }^{1}$

Theoretically, a lot of attention has been paid on the investigation of the electrical conductivity in graphene. The dc conductivity at zero and finite magnetic fields was studied theoretically by different groups. ${ }^{2-9}$ The density of states (DOS), the LL structure and the electrical conductivity were obtained from the Boltzmann transport theory and/or the Kubo formula with scattering from short- and long-range impurities that were considered in the Born or the $T$ matrix approximation. ${ }^{2-7}$ The dependence of the conductivity due to an isolated impurity located at different sites (e.g., sitelike, bondlike, or hollowlike) was further evaluated. ${ }^{8}$

The real part of the optical conductivity gives information about the electromagnetic absorption and the Raman signal which were investigated experimentally ${ }^{10-14}$ and theoretically. ${ }^{15-20}$ In the case of quantized and well separated LLs, transitions between LLs are induced which should lead to a delta-function peak structure in the absorption spectrum. However, broadened peaks were observed due to the pres- ence of disorder. ${ }^{10-14}$ Using Lorentzian fitting to the far infrared transmission spectrum, Orlita et al..$^{10}$ found that the width of the absorption peaks has a sublinear $\sqrt{B}$ dependence at high magnetic fields. Shon and $\mathrm{Ando}^{7}$ considered two different types of scatterers and found a $\sqrt{B}$ dependence for the broadening of the LLs. They included short-range scattering where the range is smaller than the lattice constant and the long-range case where the range is comparable or slightly larger than the lattice constant but still much smaller than the typical electron wavelength. In both cases the impurity scattering potential was modeled by a $\delta$ type of function. Similar experiments by Jiang et al. ${ }^{11}$ found that the LLs were strongly broadened by $20-35 \mathrm{meV}$ and it was hard to assess any $B$ dependence of the half width of the different observed resonances. Recently, Henriksen et al. ${ }^{14}$ observed a different behavior for the $n=0 \rightarrow 1$ optical transmission of exfoliated graphene and found that the cyclotron resonance (CR) peak energy exhibited a $\mathrm{V}$ shape as a function of the filling factor for $0<\nu<4$. Maximal and minimal values of the CR energy were found for, respectively, half and integer filling factors. Such a dependence on the filling factor implies that electronelectron (e-e) interaction should be important.

The width of the optical absorption at low temperature is determined by disorder. Scattering on impurities is believed to be the most important scattering mechanism determining the LL broadening at low temperature. We can distinguish two types of scattering, i.e., long-range charged impurities and short-range impurities (e.g., defects and vacancies). From the linear density dependence of the conductivity of graphene it was concluded that the most important disorder for graphene on $\mathrm{SiO}_{2}$ is due to charged impurities. ${ }^{21}$ This conclusion was seriously questioned in Ref. 22. A sublinear dependence of the conductivity on the backgate voltage was also found which was explained by invoking scattering from both long-range and short-range scatterers. ${ }^{23,24}$ The ratio $\tau_{t} / \tau_{q}$ (i.e., the transport scattering time $\tau_{t}$ to the quantum scattering time $\tau_{q}$ ) was found to have different values (i.e., 1.5 up to 5.1) for different samples which suggests that both charged impurities and short-range scatterers are active 
whose relative importance depends on the specific sample. ${ }^{24-26}$ The $T$ matrix approach ${ }^{15}$ and the Born approximation ${ }^{16}$ was used to calculate the optical conductivity at a finite magnetic field where the effect of disorder due to localized and extended defects was taken into account. Dóra and Thalmeier ${ }^{27}$ studied the DOS and magneto-optical conductivity in the presence of localized disorder and the self-energy was calculated within the full self-consistent Born approximation (SCBA) which is similar to the work of Ref. 15. In their work, they focused on the frequency and magnetic field dependence of the DOS due to localized disorder. An anomalous absorption line was found by assuming a constant LL broadening. ${ }^{17,18}$ Recently, the optical Hall conductivity in graphene and in the ordinary 2DEG was studied using the Kubo formula. ${ }^{19}$

In the present paper, we use the standard Green's-function approach to study the optical conductivity in monolayer graphene in quantizing magnetic fields. We consider the effect of scattering with both charged and short-range impurities. The scattering potential due to charged impurities is taken Coulombic which is modified by screening induced by the e-e interaction which removes the singularity at small $q$ values in the electron-charged impurity scattering potential. The magnetic field is taken sufficiently strong such that the electron energy is quantized in LLs and the impurity potential is assumed weak enough so that single impurity scattering is a good approximation. Furthermore, scattering from short-range impurities is included where the strength of this impurity potential is assumed to be independent of the wave vector. In contrast with previous works, we will calculate the LL width due to a combination of both kinds of impurity scatterers within a self-consistent Born approximation. Also, distinct from previous studies we include both intra- and inter-LL scattering effects.

The paper is organized as follows. In Sec. II the formalism to calculate the optical conductivity is given where we will use the Kubo formula. A quantizing magnetic field is applied perpendicular to the 2D plane of the graphene layer. The self-energy induced by scattering from both charged impurities and short-range impurities is evaluated from which we obtain the broadening of the LLs. We study the dependence of the DOS and the positions and strength of the different peaks in the magneto-optical conductivity on the relative contribution of the two impurity scattering mechanisms. The numerical results are presented and discussed in Sec. III and the main conclusions are summarized in Sec. IV.

\section{THEORETICAL APPROACHES}

In this paper, the graphene sheet is taken along the $x y$ plane and a uniform static perpendicular magnetic field with strength $B$ is applied. The single free carrier Hamiltonian in an external magnetic field can be solved analytically. The wave function and energy spectrum are given by ${ }^{28}$

$$
\left\langle\mathbf{r} \mid n, x_{0}\right\rangle=\psi_{n, x_{0}}(\mathbf{r})=C_{n} e^{-i y x_{0} / l_{B}^{2}}\left[\begin{array}{c}
S_{n} h_{|n|-1}\left(x-x_{0}\right) \\
h_{|n|}\left(x-x_{0}\right)
\end{array}\right],
$$

$$
E_{\alpha}=E_{n}=S_{n} \sqrt{|n|} \hbar \omega_{B} .
$$

Here, $\mathbf{r}=(x, y)$ is the spatial coordinate in the graphene plane, $C_{n}=\sqrt{\left(1+\delta_{n, 0}\right) / 2}, \alpha=\left(n, x_{0}\right)$ specifies the electronic state, $n(=0, \pm 1, \ldots)$ is the LL index, $x_{0}=k_{y} l_{B}^{2}$ is a good quantum number which connects the carrier wave vector along the $y$ direction with its average position along the $x$ direction, $l_{B}$ $=(\hbar / e B)^{1 / 2}$ is the radius of the ground cyclotron orbit, $\hbar \omega_{B}$ $=\sqrt{2} \gamma / l_{B}$ is the effective magnetic energy with $\gamma=\hbar v_{F}$ the band parameter, and $h_{n}(x)=i^{n}\left(2^{n} n ! \sqrt{\pi} l_{B}\right)^{-1 / 2} e^{-x^{2} / 2 l_{B}^{2}} H_{n}\left(x / l_{B}\right)$ with $H_{n}(x)$ the Hermite polynomial. Furthermore, $S_{n}=1$ for an electron when $n>0, S_{n}=-1$ for a hole when $n<0$, and $S_{n}=0$ for $n=0$. The LLs are degenerate with degeneracy $D_{0}=1 /\left(2 \pi l_{B}^{2}\right)$ which is proportional to the magnetic field $B$.

Now we use the wave function given above to construct the carrier quantum field operators, $\Psi^{\dagger}(\mathbf{r})=\Sigma_{\alpha} a_{\alpha}^{\dagger} \psi_{\alpha}^{*}(\mathbf{r})$ and $\Psi(\mathbf{r})=\Sigma_{\alpha} a_{\alpha} \psi_{\alpha}(\mathbf{r})$, where $a_{\alpha}^{\dagger}$ and $a_{\alpha}$ are, respectively, the creation and annihilation operators for a carrier in a LL state $|\alpha\rangle$. These field operators satisfy the anticommutation rules. The optical conductivity is given by the Kubo formula

$$
\sigma(\omega)=-\frac{i}{\hbar \omega} \frac{1}{S} \int_{0}^{\beta} d \tau e^{i \omega \tau}\left\langle T_{\tau} j(\tau) j(0)\right\rangle,
$$

where $T_{\tau}$ is the Dyson time ordering operator, $S$ is the area of the $2 \mathrm{D}$ plane, and $\beta=1 / k_{B} T$ is the inverse temperature with $k_{B}$ the Boltzmann constant. The current operator is given by

$$
j=\sum_{n, x_{0}} \sum_{n^{\prime}} e \frac{\gamma}{\hbar} C_{n} C_{n^{\prime}}\left[S_{n^{\prime}} \delta_{|n|,\left|n^{\prime}\right|-1}+S_{n} \delta_{|n|-1,\left|n^{\prime}\right|}\right] a_{n, x_{0}}^{\dagger} a_{n^{\prime}, x_{0}},
$$

which satisfies $j(\tau)=e^{\tau H} j e^{-\tau H}$ in the Heisenberg representation. To calculate the current-current correlation function, we replace the unperturbed Green's function for a carrier by the full Green's function. Due to the interaction with charged impurities and with short-range impurities, the induced selfenergy should be included within the Green's function. Taking the summation over the fermion frequency, ${ }^{29}$ the real part of the conductivity [Eq. (2)] becomes

$$
\begin{aligned}
\operatorname{Re} \sigma(\omega)= & \sigma_{0} \frac{g_{s} g_{v}}{2 \pi l_{B}^{2}} \frac{\gamma^{2}}{\hbar \omega} \sum_{n, n^{\prime}} C_{n}^{2} C_{n^{\prime}}^{2}\left(S_{n^{\prime}}^{2}, \delta_{|n|,\left|n^{\prime}\right|-1}+S_{n}^{2} \delta_{|n|-1,\left|n^{\prime}\right|}\right) \\
& \times \int_{-\infty}^{\infty} \frac{d E}{\pi}\left[n_{F}(E)-n_{F}(E+\hbar \omega)\right] \\
& \times \operatorname{Im} G_{n}(E) \operatorname{Im} G_{n^{\prime}}(E+\hbar \omega)
\end{aligned}
$$

where

$$
G_{n}(E)=\left[E-E_{n}-\Sigma_{n}(E)\right]^{-1}
$$

is the full Green's function, $\sigma_{0}=e^{2} / \hbar$ is the quantum of conductivity, and $n_{F}(E)$ is the Fermi distribution function. From Eq. (4) we find that resonant transmission occurs when the condition $E=\left|E_{|n| \pm 1}-E_{n}\right|$ is satisfied. In the Born approximation, the self-energy $\Sigma_{n}(E)$ due to the presence of charged impurities and short-range impurities is given by $\Sigma_{n}(E)$ $=\sum_{n}^{C}(E)+\sum_{n}^{S}(E)$, where 


$$
\sum_{n}^{C}(E)=\sum_{n^{\prime}} \Gamma_{n, n^{\prime}}^{2} G_{n^{\prime}}(E)
$$

with $\Gamma_{n, n^{\prime}}^{2}=n_{i}^{c} \sum_{q} C_{n, n^{\prime}}\left(l_{B}^{2} q^{2} / 2\right)\left(V_{q} e^{-q d}\right)^{2} . n_{i}^{c}$ is the charged impurity density, $\quad C_{n^{\prime} n}(x)=C_{n^{\prime}}^{2} C_{n}^{2}[N ! /(N+J) !] e^{-x} x^{J}$ $\times\left[L_{N}^{J}(x)+S_{n^{\prime}} S_{n} \sqrt{\frac{N+J}{N}} L_{N-1}^{J}(x)\right]^{2}$ is the square of the electroncharged impurity interaction matrix element with $N$ $=\min \left(\left|n^{\prime}\right|,|n|\right), \quad J=\| n^{\prime}|-| n||, \quad$ and $\quad L_{N}^{J}(x)$ the associated Laguerre polynomial. $V_{q}=2 \pi Z e^{2} / \kappa_{1} q$ is the Fourier transform of the Coulomb impurity potential, where $Z$ is the impurity charge number and $\kappa_{1}$ is the static dielectric constant for impurities in the $\mathrm{SiO}_{2}$ wafer layer. In our numerical calculation, we take $Z=1, \kappa_{1}=4.25$ and we consider charged impurities separated from the graphene layer and situated in a 2D layer at a distance $d$ from graphene. Here, in our calculation, we include the e-e screening effect through $V_{q} \rightarrow V_{q} / \epsilon(q)=2 \pi e^{2} Z /\left[\kappa_{1}\left(q+K_{q}\right)\right]$, where $\epsilon(q)$ is the static dielectric function, ${ }^{30-32} K_{q}$ is the inverse screening length calculated within the random-phase approximation (RPA) which at low temperatures is mainly determined by the intra-LL e-e interaction. In the limit of long-rang scattering, the RPA inverse screening length becomes $K_{q} \simeq$ $-\left(g_{s} g_{v} e^{2} / \pi \kappa l_{B}^{2}\right) \sum_{n} C_{n n}\left(l_{B}^{2} q^{2} / 2\right) \operatorname{Im} G_{n}\left(E_{F}\right)$, where $\kappa=2.5$ is the dielectric constant for carriers in graphene, $g_{s}$ and $g_{v}$ are the spin and valley degeneracy, and $E_{F}$ is the Fermi energy. The self-energy due to short-range scattering is given by ${ }^{7,16}$

$$
\sum_{n}^{S}(E)=\Gamma^{2} \sum_{n^{\prime}} G_{n^{\prime}}(E),
$$

where $\Gamma^{2}=\left(n_{i} V_{0}^{2} / 2\right) D_{0} \sim\left(\hbar \omega_{B}\right)^{2}$ which implies that the level broadening due to short-range scattering is proportional to $\sqrt{B} . n_{i}$ is the short-range impurity concentration and assuming the strength of the impurity potential to be a constant, we have $V=\Sigma_{i} V_{0} \delta\left(\mathbf{r}-\mathbf{r}_{\mathbf{i}}\right)$.

The total electron self-energy can be separated in a real and an imaginary parts $\Sigma_{n}(E)=\Delta_{n}(E)-i \Gamma_{n}(E)$, where the real part $\Delta_{n}(E)$ results in an energy shift and the imaginary part $\Gamma_{n}(E)$ determines the single-particle quantum level broadening of the $n$th LL. The DOS for carriers in the $n$th LL is then given by

$$
D_{n}(E)=\left(-\frac{1}{\pi}\right) g_{s} g_{v} D_{0} \operatorname{Im} G_{n}(E) .
$$

In the limit of well separated LLs and assuming that the energy is close to the $n$th LL, inter-LL scattering can be neglected. Only the term $n=n^{\prime}$ is retained in the self-energy [Eqs. (6) and (7)]. The corresponding DOS is given by

$$
D_{n}(E)=\frac{g_{s} g_{v} D_{0}}{\pi} \frac{1}{\Gamma_{n}} \operatorname{Re}\left[1-\left(\frac{E-E_{n}}{2 \Gamma_{n}}\right)^{2}\right]^{1 / 2},
$$

where $\Gamma_{n}=\Gamma_{n, n}+\Gamma$ is the single-particle quantum level broadening induced by the two kinds of impurity scattering.

From the above discussion, the optical conductivity relates to the imaginary part of the Green's function which is determined by the broadened DOS. Notice that the charged impurity scattering contribution to the LL width is determined by the screened electron charged impurity scattering potential. Because the RPA inverse screening length is deter-
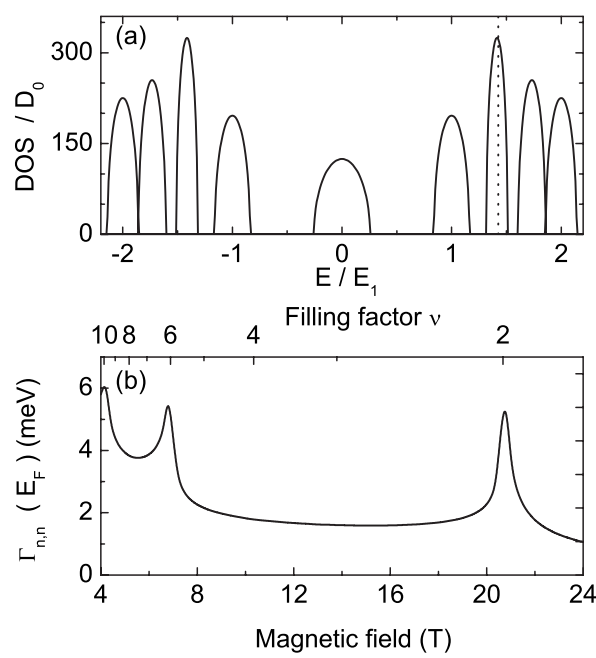

FIG. 1. (a) The DOS as a function of energy at $B=5 \mathrm{~T}$ and (b) the LL width at the Fermi energy $E_{F}$ as a function of magnetic field (or filling factor, upper axis) for a fixed electron density $n_{e}=10^{12} \mathrm{~cm}^{-2}$ and charged impurity density $n_{i}^{c}=10^{11} \mathrm{~cm}^{-2}$ limited. $D_{0}=\left(2 \pi l_{B}^{2}\right)^{-1}$ is the degeneracy of each LL. The vertical dotted line in (a) indicates the position of $E_{F}$.

mined by the imaginary part of the Green's function, the full Green's function or DOS must be evaluated self-consistently. ${ }^{33}$ From Eqs. (6) and (7), the self-energies are expressed by the squares of the intra-LL and inter-LL electron-impurity interaction matrix elements. In the limit of well separated LLs, coupling between different LLs can be ignored and intra-LL coupling is retained. If the width is comparable to or larger than the energy distance between neighboring LLs, the broadened DOS will overlap, and inter-LL coupling should be included. The width of the LLs are a function of a number of variables, e.g., the magnetic field, the electron concentration, the charged impurity and the short-range impurity density, the effective distance between the graphene layer and the charged impurities, and the short-range impurity potential.

\section{RESULTS AND DISCUSSIONS}

\section{A. Charged impurity scattering}

In this paper, the material parameters corresponding to graphene are taken as follows: the band parameter $\gamma=6.5 \mathrm{eV} \AA$ (i.e., corresponding to $v_{F} \sim 1.0 \times 10^{6} \mathrm{~m} / \mathrm{s}$ ) for graphene, the typical electron density is $n_{e} \sim 10^{12} \mathrm{~cm}^{-2}$ which can be tuned by applying a gate voltage. We assume that the charged impurities are located in the $\mathrm{SiO}_{2}$ substrate and they are separated from the graphene interface by $d \sim 1 \mathrm{~nm}$ with a typical impurity density $n_{i} \sim 10^{11} \mathrm{~cm}^{-2}$. The single-particle quantum level broadening due to shortrange scattering is taken to be $\Gamma \sim(0.05-0.1) \hbar \omega_{B} \cdot{ }^{7,16} \mathrm{In}$ our numerical calculations, we include ten adjacent LLs when calculating the inter-LL coupling.

Figure 1 shows the DOS and the LL width at the Fermi energy in the presence of only charged impurity scattering for a fixed electron density $n_{e}=10^{12} \mathrm{~cm}^{-2}$ and charged impurity density $n_{i}^{c}=10^{11} \mathrm{~cm}^{-2}$. From Fig. 1(a) we notice that 


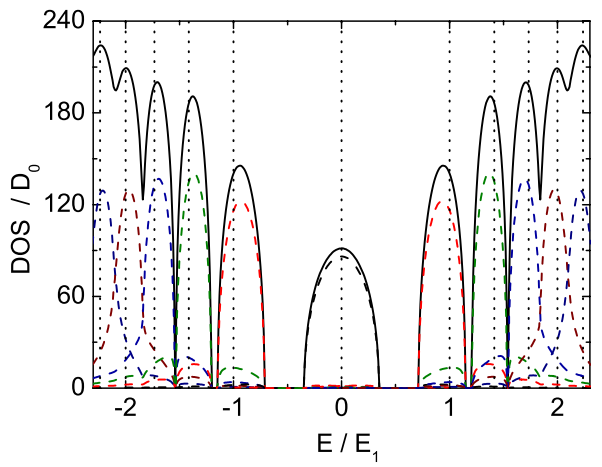

FIG. 2. (Color online) The DOS as a function of energy including inter-LL coupling for a larger charged impurity density $n_{i}^{c}=2 \times 10^{11} \mathrm{~cm}^{-2}$ in the presence of only charged impurity scattering for $B=5 \mathrm{~T}$ and $n_{e}=10^{12} \mathrm{~cm}^{-2}$. The solid black curve indicates the total DOS and the dashed color curves are the DOS of each separate LL. The vertical dotted lines indicate the positions of the nondisordered LLs. The Fermi level is located at $E_{F} / E_{1}=1.404$.

the broadened DOS are symmetric around $E=0$. The LLs are well separated for $B=5 \mathrm{~T}$ and $E / E_{1}<1.8$ and coupling between different LLs can be neglected. The peaks of the DOS are centered around the LL energies $E_{n}$. The dependence of the electron-charged impurity potential on the modulus $q$ of the momentum transfer and the oscillation of the associated Laguerre polynomial results in a sharpening of the LL at the Fermi energy. The LL width at $E_{F}$ is plotted in Fig. 1(b) and we notice that: (1) it oscillates with magnetic field or filling factor and (2) on the average it decreases with increasing magnetic field which is consistent with the results of Refs. 32 and 33. The energy spacing between subsequent LLs $(\sqrt{n+1}-\sqrt{n}) \hbar \omega_{B} \simeq \hbar \omega_{B} / 2 \sqrt{n}$ for large $n$ decreases with increasing energy [see Fig. 1(a)]. Increasing the charged impurity density, the level broadening due to charged impurities increases leading to overlapping LLs which implies that inter-LL coupling should be included. Figure 2 shows the DOS with inter-LL coupling included. It exhibits now a continuous background for $n>2$ on which the oscillating LLs are superimposed. The nonequidistant LLs result in an asymmetric inter-LL coupling which is different from the symmetric DOS in a conventional equidistant LL system. ${ }^{30}$ In Fig. 2 the peaks of the broadened DOS are shifted to lower energy as compared to the nondisordered LLs which is a consequence of this asymmetric coupling. In Ref. 27, asymmetric peaks in the DOS were attributed to the self-energy due to localized impurities which was obtained within the full SCBA. Therefore, the type of scattering and the used approximation when calculating the self-energy determine the shape of the broadened DOS and modify the position of the LLs.

From Fig. 1(b), it may appear strange that the LL width on average decreases with increasing magnetic field. The width of the electron wave function is proportional to $l_{B} \sim 1 / \sqrt{B}$ and for scattering on Coulomb impurities, $V(r) \sim 1 / r$, one expects that the LL width should increase as $\sqrt{B}$. The origin of the different $B$ dependence can be traced back to the screening effect. Therefore, we repeated the calculation and replaced the inverse screening length $K_{q}$ by a

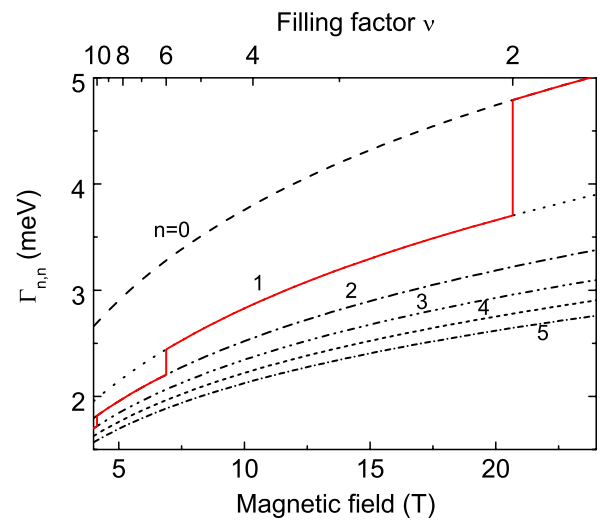

FIG. 3. (Color online) The LL width as a function of magnetic field in the presence of only charged impurity scattering with magnetic field independent Thomas-Fermi screening wave vector $q_{\mathrm{TF}}$. The solid red curve indicates the LL width at the Fermi energy. The parameters are the same as in Fig. 1.

constant value $q_{\mathrm{TF}}$ using a Thomas-Fermi (TF) screening wave vector independent of the magnetic field. The numerical results are shown in Fig. 3 and exhibit the expected $\sqrt{B}$ dependence. It is the magnetic field dependence of the screening which leads to the very different $B$ dependence of the broadening shown in Fig. 1(b). For completeness we show the screened and bare Coulomb potentials in Fig. 4 for the same conditions as in Fig. 1 and $B=5$ T. Using Fourier transform, we obtain the Coulomb potential in real space (see inset of Fig. 4). Notice that the screened Coulomb potential $V(r)$ is reduced as compared to the bare potential which is a consequence of the strong screening at small $q$ values.

\section{B. Short-range impurity scattering}

The DOS including only short-range impurity scatterers is shown in Fig. 5. For an intermediate value of the singleparticle broadening induced by short-range scattering $\Gamma=0.1 E_{1}, 7,16$ the full width at half maximum is $2 \Gamma=0.2 E_{1}$. Only the broadened DOS for the $n=0,1$ LLs is clearly sepa-

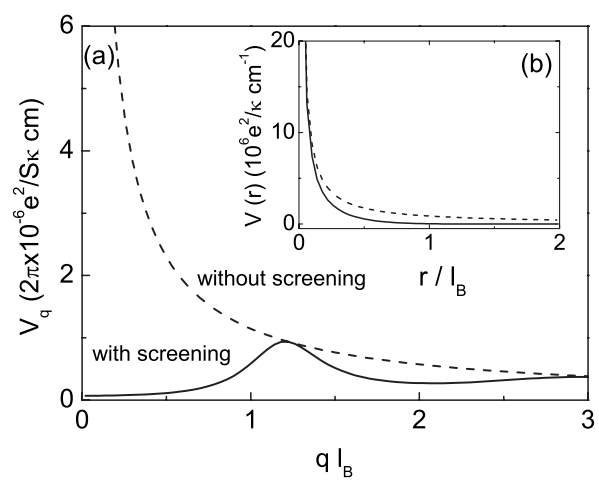

FIG. 4. The Fourier transform of the bare (dashed lines) and screened (solid lines) Coulomb potential in the presence of only charged impurity scattering and intra-LL screening $K_{q}$. The inset shows the potential in real space. The parameters are the same as in Fig. 1 and $B=5 \mathrm{~T}$. 


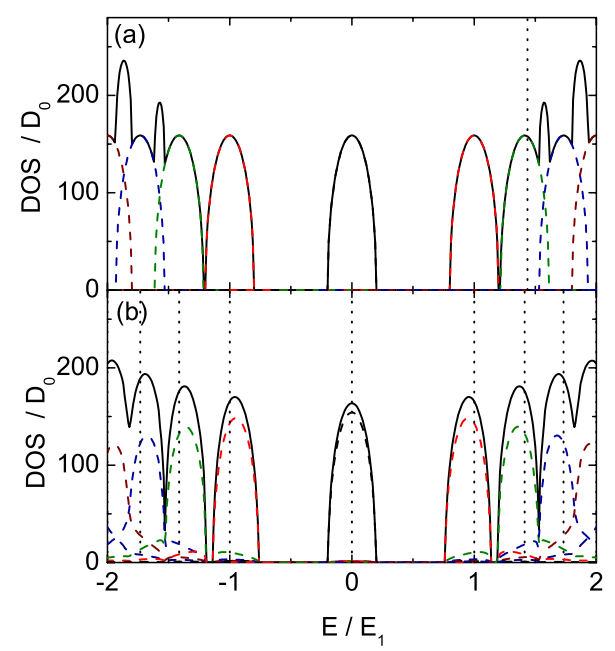

FIG. 5. (Color online) The DOS as a function of energy (a) without and (b) with inter-LL coupling in the presence of only short-range scattering at $B=5 \mathrm{~T}$. The solid black curves in (a) and (b) indicate the total DOS and the dashed color curves are the DOS of each LL. The vertical dotted lines in (a) and (b) indicate, respectively, the positions of the Fermi energy and the nondisordered LLs. The Fermi level in (b) is located at $E_{F} / E_{1}=1.436$ when $n_{e}=10^{12} \mathrm{~cm}^{-2}$ and is shown by the dotted vertical line in (a).

rated while the overlap between the other adjacent LLs is obvious in Fig. 5(a) which leads to spurious sharp peaks between the LLs. Therefore, we included inter-LL coupling with the adjacent 10 LLs [i.e., including $n^{\prime}=n, n^{\prime}$ $=n \pm 1, \ldots, n^{\prime}=n \pm 10$ in the self-energy $\sum_{n}^{S}(E)$ of Eq. (7)] and obtain the DOS plotted in Fig. 5(b). Now the DOS exhibits only peaks near the LL energies [Eq. (1b)]. The LLs $n=0$ and $n=1$ are broadened and well separated in the Born approximation.

\section{Charged impurity and short-range impurity scattering}

The DOS, in the presence of both charged impurity and short-range impurity scattering, is plotted in Fig. 6 where we included inter-LL coupling. At a fixed electron density $n_{e}=10^{12} \mathrm{~cm}^{-2}$ and magnetic field $B=5 \mathrm{~T}$, the filling factor $\nu=8.27$ and the $n=2 \mathrm{LL}$ is partially filled. The width of each LL is determined self-consistently and includes contributions from long- and short-range scattering. Larger broadening is obtained with increasing single-particle quantum level broadening due to short-range impurities or by increasing the charged impurity density in which case inter-LL coupling becomes more important. The broadened DOS at integer filling $\nu=10$ is plotted in Fig. 7. Filling factor $\nu=10$ corresponds to a Fermi level position between the $n=2$ and $n=3$ LLs. Here, due to the broadening of LLs, the self-consistent Fermi energy is located in the broadened $n=3$ LL which implies that most states for the $n=2 \mathrm{LL}$ are occupied and the $n=3$ LL is only filled by a few electrons. Because of the small screening the LLs are more broadened.

Figure 8 shows the LL width at the Fermi energy as a function of magnetic field including charged impurity and short-range scattering, where results are shown for three different values of the strength of short-range impurity scatter-

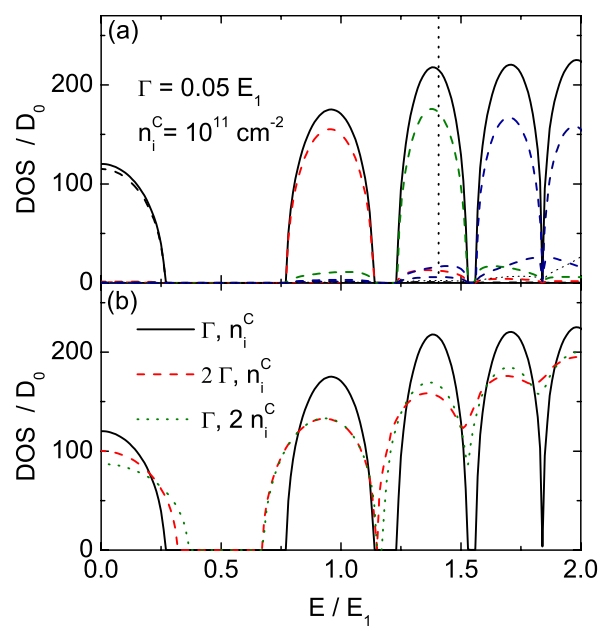

FIG. 6. (Color online) The DOS as a function of energy for different charged impurity density and single-particle level broadening due to short-range impurities when $n_{e}=10^{12} \mathrm{~cm}^{-2}$ and $B=5 \mathrm{~T}(\nu=8.27)$. The solid black curves in (a) indicate the total DOS and the dashed color curves are the DOS of each LL. The vertical dotted line in (a) indicates the position of the Fermi energy $E_{F}$.

ing. From Fig. 8 we note that: first, by including two types of scatterers the single-particle LL broadening increases slowly with increasing magnetic field near half filling which can be fitted (dashed-dotted-dotted curves) to a $B^{\alpha}$ functional dependence with $\alpha<0.5$ which is consistent with the found experimental dependence. ${ }^{10}$ Second, increasing the strength of short-range scattering, $\alpha$ increases because of the larger contribution to the Level broadening from short-range impurities. Meanwhile, stronger inter-LL coupling leads to a larger superposition of LLs. More broadened DOS results in a weaker oscillatory behavior of the RPA screening length and smaller oscillations of the total LL width with filling factor. Third, it should be noted that the LL width from charged impurities oscillates as a function of the filling factor. At integer filling, the electron screening effect is weak and the LL width is enhanced.

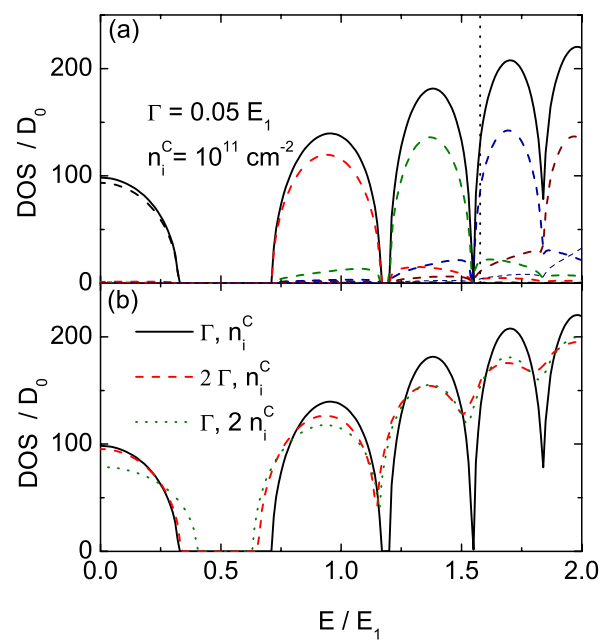

FIG. 7. (Color online) The same as Fig. 6 but now for integer filling $\nu=10$. 


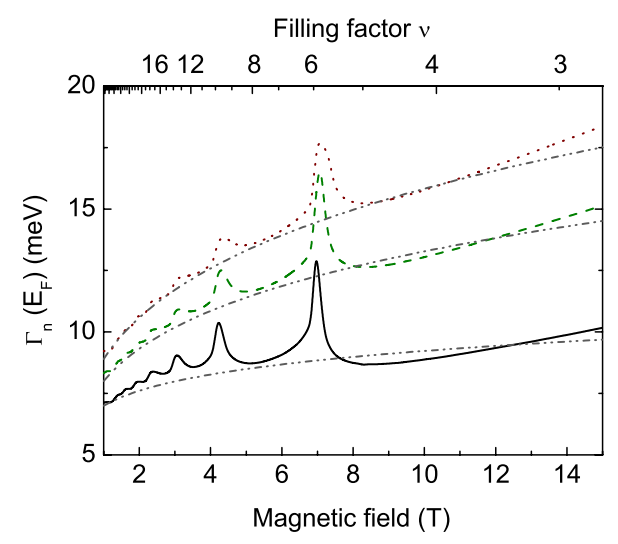

FIG. 8. (Color online) The LL width at $E_{F}$ as a function of magnetic field in the presence of both charged impurity and shortrange impurity scattering including inter-LL coupling. Solid black, dashed olive, and dotted wine curves are for different strength of short-range broadening $\Gamma / E_{1}=0.05,0.08$ and 0.1 , respectively. The dashed-dotted-dotted dark gray curves fit the width near half filling with $\sim B^{\alpha}$ where $\alpha=0.12,0.22$, and 0.25 , respectively. The other parameters $n_{e}$ and $n_{i}^{c}$ are the same as in Fig. 1(a).

\section{Magneto-optical conductivity}

Figure 9 shows the real part of the optical conductivity as a function of photon energy at a fixed magnetic field. Several absorption peaks can be observed. We know that for a conventional 2DEG $E_{n+1}-E_{n}=\hbar \omega_{c}$ so that only one absorption peak is typically observed with frequency $\omega=\omega_{c}$. In contrast, for graphene because $E_{n^{\prime}}-E_{n}$ depends on the LL index $\sqrt{|n|}$ [see Eq. (1b)], the selection rule $\delta_{\left|n^{\prime}\right|=|n| \pm 1}$ can give rise to a series of peaks in the magneto-optical conductivity. At a given magnetic field $B=5 \mathrm{~T}$ and electron density $n_{e}=10^{12} \mathrm{~cm}^{-2}$, the $n=2 \mathrm{LL}$ is partially filled which implies

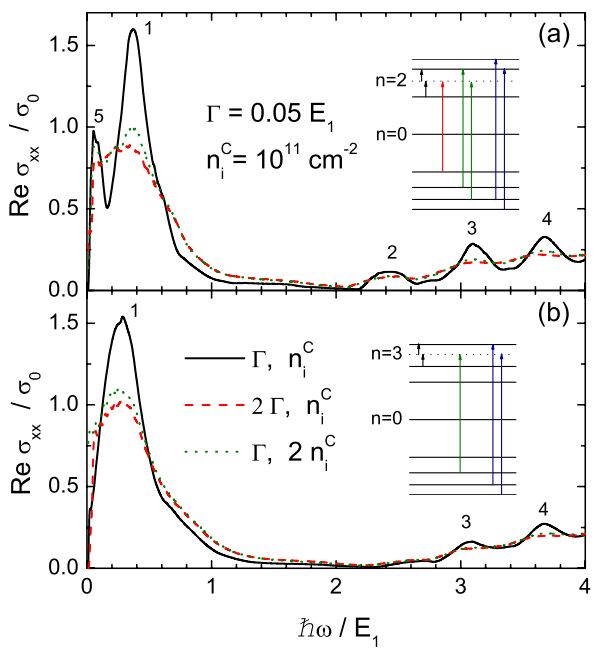

FIG. 9. (Color online) The optical conductivity as a function of photon energy at $B=5 \mathrm{~T}$ for two values of the filling factor (a) $\nu$ $=8.27\left(n_{e}=10^{12} \mathrm{~cm}^{-2}\right)$ and (b) $\nu=10$ for different single-particle quantum level broadening induced by charged impurities and shortrange impurities. The insets show a schematic LL ladder with allowed transitions indicated by arrows and the horizontal dotted line indicates the position of the Fermi energy.

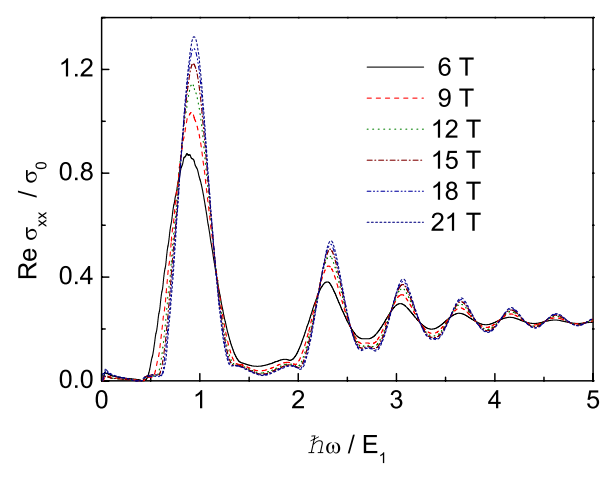

FIG. 10. (Color online) The optical conductivity as a function of photon energy for fixed filling factor $\nu=2$ for different magnetic fields at $n_{i}^{c}=10^{11} \mathrm{~cm}^{-2}$ and $\Gamma=0.08 E_{1}$.

that the Fermi level is situated in the $n=2 \mathrm{LL}$. The intraband transitions from $E_{2}$ to $E_{3}$ and from $E_{1}$ to $E_{2}$ contribute to the broad peak " 1 " due to their almost equal energy spacing. The intensity of these intraband transitions is large as compared to the intensity of the other interband transitions. The peak " 2 " is caused by the first interband transition from $E_{-1}$ to $E_{2}$. Two interband transitions result in the two other peaks which have almost twice the oscillator strength of the previous peak of the interband transition (e.g., the peak " 3 " comes from the transitions from $E_{-2}$ to $E_{3}$ and $E_{-3}$ to $\left.E_{2}\right)$. All the interband transitions except the first one appear in pairs. All allowed transitions are plotted in the inset of Fig. 9(a). Interestingly, around the Fermi energy, adjacent filled LLs due to inter-LL coupling (see Fig. 6) leads to extra transitions. Notice, a small sharp peak " 5 " is found for small frequency which is the result of this particular value of the filling factor. This result differs from the case when a constant broadening is assumed as in Refs. 17 and 18. The reason is that, in their calculation, no LL mixing was taken into account. When increasing the broadening the peaks in the absorption spectrum are smoothened and some of them disappear.

The magneto-optical transition via absorption scattering occurs mainly through electronic transitions from lower occupied LLs to higher empty LLs. Different transition channels have different transition probabilities [see Eq. (4)]. The filling of the LLs decides the possible transitions that contribute to the optical absorption. When increasing the electron density $n_{e}$, the filling factor increases. In Fig. 9(b), the filling factor is increased to $\nu=10$ for $B=5 \mathrm{~T}$, which shifts the Fermi energy to the $n=3 \mathrm{LL}$. Now the intraband transitions from $E_{3}$ to $E_{4}$ and from $E_{2}$ to $E_{3}$ contribute to the first broadened peak which shifts the position of this peak to lower energy. The first interband transition induced by the transition from $E_{-1}$ to $E_{2}$ shown in Fig. 9(a) is now no longer possible. The intensity of the peak 3 halves because only the transition from $E_{-2}$ to $E_{3}$ contributes to it. The rest of the peaks remain roughly unchanged. The corresponding transition channels are given in the inset of Fig. 9(b). The above optical conductivity results from the electronic transitions with the selection rules $\left|n^{\prime}\right|=|n| \pm 1$ and can be explained from the broadened LLs shown in Figs. 6 and 7.

Figure 10 shows the optical conductivity in units of $\sigma_{0}$ $=e^{2} / \hbar$ as a function of $\hbar \omega / E_{1}$ at a fixed filling factor $\nu=2$ for 

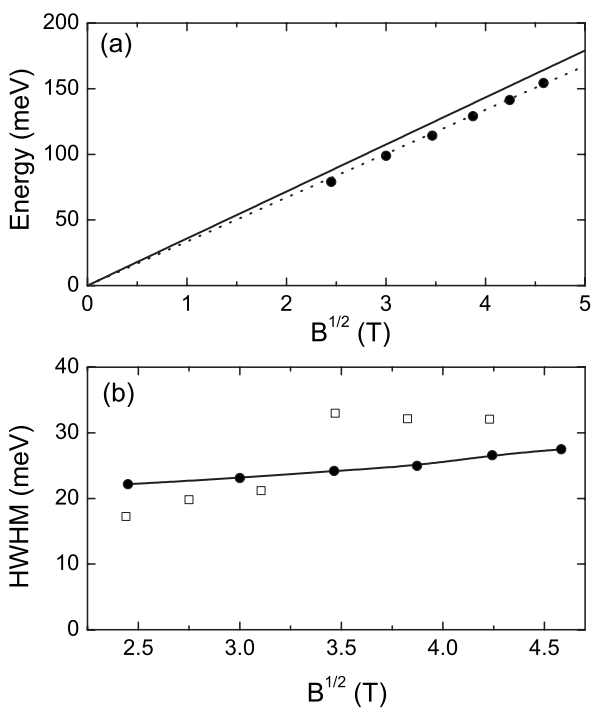

FIG. 11. (a) The peak position (full circle) and (b) the HWHM (-) of the $n=0 \rightarrow 1$ transition vs $\sqrt{B}$. The solid line in (a) corresponds to the $n=1$ single-particle Landau level. The open square symbols in (b) are the experiment results from Ref. 11. The parameters are the same as in Fig. 10.

different magnetic field. Notice that: first, at a filling factor of $\nu=2$ the $n=0 \rightarrow 1$ transition results in the first strong peak. Comparing with Ref. 15 where scattering only from shortrange impurities was included, the self-consistent DOS induced by electron-impurity (e-i) scattering using the $T$ matrix approximation showed a large broadening around zero energy which smeared the $n=0, \pm 1$ LLs into a single broadened peak. Their analysis showed that the transitions involving the $n=0 \mathrm{LL}$ were suppressed which does not agree with our findings. The transition involving the $n=0 \mathrm{LL}$ has been observed in far infrared transmission experiments. Second, the optical conductivity is proportional to the degeneracy $\left(D_{0} \sim B\right)$ and $\omega_{B}$ is proportional to $\sqrt{B}$ which leads to an increased oscillator strength at high magnetic field. Third, the peak positions of the LLs in the presence of disorder are slightly shifted to lower energy. The peak energy of the $n$ $=0 \rightarrow 1$ transition is plotted in Fig. 11(a) which is a little lower than the $n=1 \mathrm{LL}$ energy. Fourth, the half width at half maximum (HWHM) of the optical conductivity in Fig. 11(b) is consistent with the experiment results but notice that the experimental results ${ }^{11}$ exhibit some extra structure which is not reproduced by our theory.

For a fixed magnetic field $B=18 \mathrm{~T}$, we varied the electron density around the filling factor $\nu=2$ and plotted the optical conductivity in Fig. 12. A notable feature is that the peak position of the $n=0 \rightarrow 1$ transition shows a "V" shape as a function of the filling factor around $\nu=2$. The reason is that in the presence of charged impurity scattering, the level broadening is determined by the screened electron-charged impurity potential. The RPA screening length is determined by the imaginary part of the Green's function at the Fermi energy which oscillates with magnetic field (or filling factor). At integer filling $\nu=2$, the screening is weak which gives rise to a large broadening of the DOS [see Fig. 12(b)] for the $n$ $=1 \mathrm{LL}$ and a large shift of the renormalized LLs. Such a

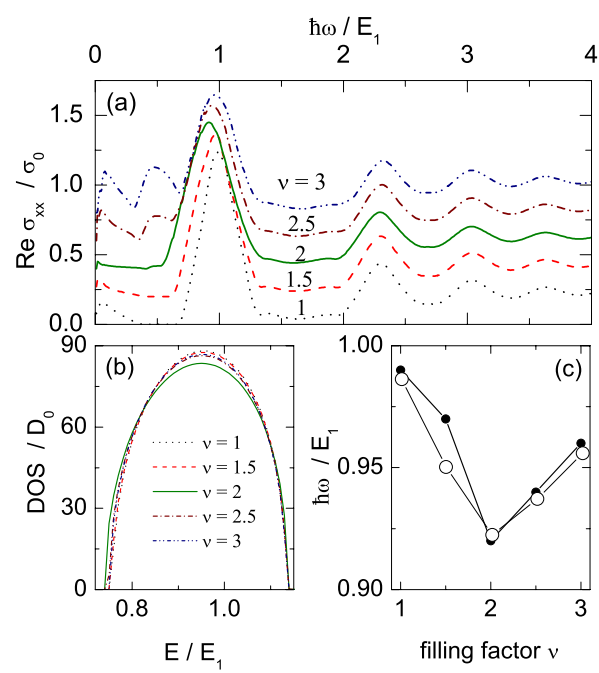

FIG. 12. (Color online) (a) The optical conductivity as a function of photon energy at a fixed magnetic field $B=18$ T for different filling factor with $n_{i}^{c}=10^{11} \mathrm{~cm}^{-2}$ and $\Gamma=0.1 E_{1}$. Traces are offset for clarity. (b) The $n=1$ broadened DOS for the corresponding filling factor and (c) the peak position (-) of the $n=0 \rightarrow 1$ transition as a function of the filling factor. The - $\bigcirc$ - symbols are the experiment results from Ref. 14.

shifted CR peak energy with a V shape has been observed in a recent infrared transmission experiment. ${ }^{14}$ Additionally, the intensity of the peak around $0.4 E_{1}$ which corresponds to the transition $n=1 \rightarrow 2$ for $\nu>2$ is lower than that of the corresponding peak for the $n=0 \rightarrow 1$ transition. This agrees with the experimental result (see Fig. 1 in Ref. 12).

\section{CONCLUSION}

In this paper, we analyzed the effect of two types of scattering, i.e., charged and short-range impurities, on the LL broadening and the magneto-optical conductivity of graphene in the presence of a quantizing magnetic field. Moreover, contributions of both intra- and inter-LL scattering in the self-energy were taken into account. A Green'sfunction approach was employed to calculate the DOS and the self-energy within a self-consistent Born approximation. The LL widths are a function of impurity density, short-range impurity potential and filling factor. We found that: (1) the broadened DOS for the $\pm n$ LLs in the presence of disorder induced by impurities is symmetric around $E=0$. When coupling between different LLs is included, the positions of the renormalized LLs are shifted to lower energy and the LL peak structure in the broadened DOS becomes less pronounced. (2) In the presence of only charged impurity scattering, intra-LL e-i coupling and intra-LL e-e screening are retained in the self-energy calculation. The width of each LL oscillates as a function of the filling factor. The LL width $\Gamma_{n, n}$ at the Fermi energy exhibits maxima at integer filling. For half filling the LL width at the Fermi energy decreases monotonically with increasing magnetic field which is very different from the case when only short-range scattering is present where the LL width increases as $\sqrt{B}$. Due to the separation in energy between the subsequent LLs which be- 
haves as $\hbar \omega_{B} / 2 \sqrt{n}$ for large $n$, the broadened DOS tends to overlap which implies that inter-LL coupling should be included. The $n=0,1$ broadened DOS is separated from the other LLs within the Born approximation which is different from the result as obtained using a $T$ matrix approximation. (3) The DOS are strongly broadened in the presence of the two kinds of scattering. A $B^{\alpha}$ dependence with $\alpha<1 / 2$ is found near half filling. (4) The broadened LLs and the filling factor determine the position and intensity of the peaks in the optical conductivity. The oscillator strength for intraband transitions is much larger than that for interband transitions. The HWHM is determined by the type of scattering and the strength of disorder. (5) In a high magnetic field, transitions involving the $n=0 \mathrm{LL}$ are very pronounced which is different from the case with only short-range scattering using the $T$ matrix approximation. The found peak position and the
HWHM of the $n=0 \rightarrow 1$ transition are quantitatively consistent with experiment. (6) Due to the filling factor dependence of the LL broadening, the CR peak energy exhibits a V shape as a function of the filling factor which agrees with the observed CR transition in graphene. From our numerical results, we conclude that the LL broadening is dominated by both charged impurities and short-range impurities.

\section{ACKNOWLEDGMENTS}

This work was supported by the National Natural Science Foundation of China under Grant No. 10804053, the Flemish Science Foundation (FWO-V1), the Belgian Science Policy (IAP), and the Chinese Academy of Sciences and Department of Science and Technology of Yunnan Province. *francois.peeters@ua.ac.be

${ }^{1}$ K. S. Novoselov, A. K. Geim, S. V. Morozov, D. Jiang, M. I. Katsnelson, I. V. Grigorieva, S. V. Dubonos, and A. A. Firsov, Nature (London) 438, 197 (2005); Y. Zhang, Y.-W. Tan, H. L. Stormer, and P. Kim, ibid. 438, 201 (2005).

${ }^{2}$ K. Nomura and A. H. MacDonald, Phys. Rev. Lett. 98, 076602 (2007).

${ }^{3}$ E. H. Hwang, S. Adam, and S. Das Sarma, Phys. Rev. Lett. 98, 186806 (2007).

${ }^{4}$ E. H. Hwang and S. Das Sarma, Phys. Rev. B 79, 165404 (2009).

${ }^{5}$ H. M. Dong, W. Xu, Z. Zeng, T. C. Lu, and F. M. Peeters, Phys. Rev. B 77, 235402 (2008).

${ }^{6}$ T. Fukuzawa, M. Koshino, and T. Ando, J. Phys. Soc. Jpn. 78, 094714 (2009).

${ }^{7}$ N. H. Shon and T. Ando, J. Phys. Soc. Jpn. 67, 2421 (1998).

${ }^{8}$ F. M. D. Pellegrino, G. G. N. Angilella, and R. Pucci, Phys. Rev. B 80, 094203 (2009).

${ }^{9}$ W. Xu, H. M. Dong, L. L. Li, J. Q. Yao, P. Vasilopoulos, and F. M. Peeters, Phys. Rev. B 82, 125304 (2010).

${ }^{10}$ M. Orlita, C. Faugeras, P. Plochocka, P. Neugebauer, G. Martinez, D. K. Maude, A.-L. Barra, M. Sprinkle, C. Berger, W. A. de Heer, and M. Potemski, Phys. Rev. Lett. 101, 267601 (2008).

${ }^{11}$ Z. Jiang, E. A. Henriksen, L. C. Tung, Y. J. Wang, M. E. Schwartz, M. Y. Han, P. Kim, and H. L. Stormer, Phys. Rev. Lett. 98, 197403 (2007).

${ }^{12}$ M. L. Sadowski, G. Martinez, M. Potemski, C. Berger, and W. A. de Heer, Phys. Rev. Lett. 97, 266405 (2006).

${ }^{13}$ R. S. Deacon, K.-C. Chuang, R. J. Nicholas, K. S. Novoselov, and A. K. Geim, Phys. Rev. B 76, 081406(R) (2007).

${ }^{14}$ E. A. Henriksen, P. Cadden-Zimansky, Z. Jiang, Z. Q. Li, L.-C. Tung, M. E. Schwartz, M. Takita, Y.-J. Wang, P. Kim, and H. L. Stormer, Phys. Rev. Lett. 104, 067404 (2010).

${ }^{15}$ N. M. R. Peres, F. Guinea, and A. H. Castro Neto, Phys. Rev. B 73, 125411 (2006).

${ }^{16}$ T. Morimoto, Y. Hatsugai, and H. Aoki, Phys. Rev. B 78,
073406 (2008).

${ }^{17}$ V. P. Gusynin, S. G. Sharapov, and J. P. Carbotte, Phys. Rev. Lett. 98, 157402 (2007).

${ }^{18}$ V. P. Gusynin, S. G. Sharapov, and J. P. Carbotte, J. Phys.: Condens. Matter 19, 026222 (2007).

${ }^{19}$ T. Morimoto, Y. Hatsugai, and H. Aoki, Phys. Rev. Lett. 103, 116803 (2009).

${ }^{20}$ C. Zhang, L. Chen, and Z. S. Ma, Phys. Rev. B 77, 241402 (2008).

${ }^{21}$ Y.-W. Tan, Y. Zhang, K. Bolotin, Y. Zhao, S. Adam, E. H. Hwang, S. Das Sarma, H. L. Stormer, and P. Kim, Phys. Rev. Lett. 99, 246803 (2007).

${ }^{22}$ L. A. Ponomarenko, R. Yang, T. M. Mohiuddin, M. I. Katsnelson, K. S. Novoselov, S. V. Morozov, A. A. Zhukov, F. Schedin, E. W. Hill, and A. K. Geim, Phys. Rev. Lett. 102, 206603 (2009).

${ }^{23}$ C. Jang, S. Adam, J.-H. Chen, E. D. Williams, S. Das Sarma, and M. S. Fuhrer, Phys. Rev. Lett. 101, 146805 (2008).

${ }^{24}$ X. Hong, K. Zou, and J. Zhu, Phys. Rev. B 80, 241415(R) (2009).

${ }^{25}$ E. H. Hwang and S. Das Sarma, Phys. Rev. B 77, 195412 (2008).

${ }^{26} \mathrm{M}$. Monteverde, C. Ojeda-Aristizabal, R. Weil, K. Bennaceur, M. Ferrier, S. Guéron, C. Glattli, H. Bouchiat, J. N. Fuchs, and D. L. Maslov, Phys. Rev. Lett. 104, 126801 (2010).

${ }^{27}$ B. Dóra and P. Thalmeier, Phys. Rev. B 76, 035402 (2007).

${ }^{28}$ See, e.g., T. Ando, J. Phys. Soc. Jpn. 76, 024712 (2007).

${ }^{29}$ A. Ron and N. Tzoar, Phys. Rev. 131, 12 (1963).

${ }^{30}$ W. Xu and P. Vasilopoulos, Phys. Rev. B 51, 1694 (1995).

${ }^{31}$ R. Roldán, M. O. Goerbig, and J.-N. Fuchs, Semicond. Sci. Technol. 25, 034005 (2010).

${ }^{32}$ K. Nomura and A. H. MacDonald, Phys. Rev. Lett. 96, 256602 (2006).

${ }^{33}$ C. H. Yang, F. M. Peeters, and W. Xu, Phys. Rev. B 82, 075401 (2010). 